\title{
The generalized successive approximation and Padé approximants method for solving an elasticity problem of based on the elastic ground with variable coefficients
}

\author{
Mustafa Bayram
}

Department of Computer Engineering, Faculty of Engineering and Architecture, Istanbul Gelisim University, Avcilar, Istanbul, Turkey

\begin{abstract}
In this study, we have applied a generalized successive numerical technique to solve the elasticity problem of based on the elastic ground with variable coefficient. In the first stage, we have calculated the generalized successive approximation of being given BVP and in the second stage we have transformed it into Padé series. At the end of study a test problem has been given to clarify the method.
\end{abstract}

Keywords: The generalized successive approximation method, Integral Equations, BVPs, Padé series.

\section{Introduction}

The solution of BVPs has a lot of methods in literature. One of the most known is the integral equations method. By using the mentioned method, we can achieve an integral equation which is equivalent to the BVP. It is well known that the solution of the integral equation can be defined as the solution of the BVPs. This equation is generally known as Fredholm equation in the mathematical society. But in our paper we acquire a Fredholm-Volterra integral equation different from the known as so far.

The elasticity problem based on the elastic ground with variable coefficients has the following form;

$$
\begin{gathered}
\frac{d^{4} x}{d t^{4}}+a(t) x=f(t),(0 \leq t \leq T) \\
\frac{d^{2} x(0)}{d t^{2}}=A_{1}, \frac{d^{3} x(0)}{d t^{3}}=B_{1} \\
x(T)=A_{2}, \frac{d x(T)}{d t}=B_{2}
\end{gathered}
$$

where $a(t)$ and $f(t)$ are previously given continuous functions on the interval $0 \leq t \leq T$. At first, the successive approximations method has been applied to the problem and then converted to Padé series [3, 4].

\section{The equivalent integral equation}

The following linear equations

$$
x(t)=f(t)+\int_{0}^{t} K(t, s) x(s) d s
$$




$$
\begin{gathered}
x(t)=f(t)+\int_{0}^{T} K(t, s) x(s) d s \\
x(t)=f(t)+\int_{0}^{t} K_{1}(t, s) x(s) d s+\int_{0}^{T} K_{2}(t, s) x(s) d s
\end{gathered}
$$

are known in the literature as Volterra, Fredholm and Volterra-Fredholm integral equations, respectively. We call the function $f(t)$ as free term of (4)-(5), $K(t, s)$ and $K_{i}(t, s)$ are kernels of equations (4)-(5), and $x(t)$ is an unknown function defined on the interval $0 \leq t \leq T$.

Let $C[0, T]$ be the space which contains all of the continuous functions defined on the closed interval $[0, T]$. In this space the norm of $x(t) \in C[0, T]$ is a real function and given as follows,

$$
\|x\|=\max _{0 \leq t \leq T}|x(t)|
$$

We can define $F_{x}$ and $V_{x}$ as

$$
F_{x} \equiv \int_{0}^{T} K(t, s) x(s) d s
$$

and

$$
V_{x} \equiv \int_{0}^{t} K(t, s) x(s) d s
$$

on the $C[0, T]$, and the above operators are called as Fredholm and Volterra operators. If $F_{x} \in C[0, T]$ for $x(t) \in C[0, T]$, then it is said that operator $F_{x}$ affects $C[0, T]$.

If the operator $F_{x}$ acts from $C[0, T]$ to $R$ then we call the operator $F_{x}$ as linear functional. We define degenerated kernel function as the following

$$
K(t, s)=\sum_{i=1}^{n} a_{i}(t) b_{i}(s)
$$

If kernel function is degenerated, the integral equations which has this sort of kernel are known as integral equations with a degenerated kernel [5].

Let equation (4) has kernel (7) then the equation (4) can be arranged as

$$
x(t)=f(t)+\sum_{i=0}^{n} a_{i}(t) \int_{0}^{T} b_{i}(s) x(s) d s .
$$

Here, we search the solution of the eq. (8) as follows

$$
x(t)=f(t)+\sum_{i=0}^{n} a_{i}(t) C_{i}
$$

To get $C_{j}$, we can arrange a system as follows

$$
C_{i}=\int_{0}^{T} b_{i}(s) f(s) d s+\sum_{j=0}^{n} \int_{0}^{T} a_{i}(s) b_{i}(s) f(s) d s C_{j}(\mathrm{i}=1, \cdots, \mathrm{n})
$$


Let $\Delta$ be the determinant of above system. If $\Delta \neq 0$, then we find out

$$
C_{i}=\frac{1}{\Delta} \sum_{j=1}^{n} \Delta_{i j} \int_{0}^{T} b_{j}(s) f(s) d s
$$

where $\Delta_{i j}$ is known as algebraic complement of $\Delta$. The $\Delta_{i j}$ can be constructed by deleting ith row and $j$ th column of the $\Delta$. If equation (4) has degenerated kernel, the solution of the eq. (4) will be

$$
x(t)=f(t)+\sum_{i, j=1}^{n} a_{i}(t) \frac{\Delta_{i j}}{\Delta} \int_{0}^{T} b_{j}(s) f(s) d s
$$

or

$$
x(t)=f(t)+\int_{0}^{T}\left[\sum_{i, j=1}^{n} a_{i}(t) b_{j}(s) \frac{\Delta_{i j}}{\Delta}\right] f(s) d s .
$$

\section{The green functions and solution of BVPs}

We are considering the following BVPs

$$
\begin{aligned}
& x^{\prime \prime}(t)+b(t) x^{\prime}+a(t) x=f(t), 0 \leq t \leq T \\
& a_{0} x(0)+\beta_{0} x^{\prime}(0)=\gamma_{0} \\
& a_{1} x(0)+\beta_{1} x^{\prime}(0)=\gamma_{1} .
\end{aligned}
$$

where $a(t), b(t)$ and $f(t)(0 \leq t \leq T)$ are previously defined functions. Let $\alpha_{i}, \beta_{i}$ and $\gamma_{i}(i=0,1)$ are constants [6]. We convert the equation (9) into its homogeneous form as following

$$
\begin{aligned}
& x^{\prime}+b(t) x^{\prime}+a(t) x=0,(0 \leq t \leq T), \\
& a_{0} x(0)+\beta_{0} x^{\prime}(0)=0, \\
& \alpha_{1} x(0)+\beta_{1} x^{\prime}(0)=0 .
\end{aligned}
$$

Definition 1. Let $G(t, s)$ be function which has the following properties with its known value $s \in(0, T)$

(I) If $t \neq s$, then $G(t, s)$ is solution of the given problem (10).

(II) If $t=s$, then $G(t, s)$ is continuous function with respect to $t$. Partial derivative of the $G(t, s)$ with respect to thas first kind of discontinuity and its jumping number 1 .

That is,

$$
\begin{aligned}
& G(s+0, s)=G(s-0, s), \\
& G^{\prime}(s+0, s)-G_{t}^{\prime}(s-0, s)=1 .
\end{aligned}
$$

Now, we are going to construct the Green function: Let we think eq. (10) has two distinct solution such as $x_{1}(t), x_{2}(t)$ respectively and satisfies the boundary conditions (11) and (12), respectively.

Let consider the following function

$$
G(x, s)=\left\{\begin{array}{l}
\varphi(s) x_{1}(t), 0 \leq t \leq s \\
\psi(s) x_{2}(t), s \leq t \leq T .
\end{array}\right.
$$

Now we choose the functions $\varphi(t)$ and $\psi(t)$ which satisfy (3.5). That is,

$$
\psi(s) x_{2}(s)=\varphi(s) x_{1}(s), \psi(s) x_{2}^{\prime}(s)-\varphi(s) x_{1}^{\prime}(s)=1 .
$$

If we solve the above system we can get the functions $\varphi(s)$ and $\psi(s)$. By substituting $\varphi(s)$ and $\psi(s)$ in (14) we get the function $G(x, s)$ as Green function of the problem (10)-(12). 
Theorem 1. Let $G(x, s)$ be the Green functions of the problem (10)-(12) and let $f(t)$ be a continuous function, the following function

$$
x(t)=\int_{0}^{T} G(t, s) f(s) d s
$$

will be the solution for nonhomogeneous problem (9) [6].

\section{The equivalent Fredholm Volterra integral equations}

Let $F(t)=f(t)-a(t) x$. When we consider the boundary conditions (2) and the equation

$$
\frac{d^{4} x}{d t^{4}}=F(t)
$$

has integral order of four, on the interval $[0, t]$, the following equations can be arrived

$$
\begin{aligned}
& x^{\prime \prime \prime}(t)=x^{\prime \prime \prime}(0)+\int_{0}^{t} F(s) d s, \\
& x^{\prime \prime}(t)=x^{\prime \prime}(0)+x^{\prime \prime \prime}(0) t+\int_{0}^{t}(t-s) F(s) d s, \\
& x^{\prime}(t)=x^{\prime}(0)+x^{\prime \prime}(0) t+\frac{x^{\prime \prime \prime}(0) t^{2}}{2}+\int_{0}^{t} \frac{(t-s)^{2}}{2} F(s) d s, \\
& x(t)=x(0)+x^{\prime}(0) t+\frac{x^{\prime \prime}(0) t^{2}}{2}+\frac{x^{\prime \prime \prime}(0) t^{3}}{6}+\int_{0}^{t} \frac{(t-s)^{3}}{6} F(s) d s
\end{aligned}
$$

where

$$
x(t)=x(0)+x^{\prime}(0) t+\frac{A_{1} t^{2}}{2}+\frac{B_{1} t^{3}}{6}+\int_{0}^{t} \frac{(t-s)^{3}}{6} F(s) d s
$$

In addition to this, the boundary conditions (2), (3) and $x(t), x^{\prime}(t)$ have been used,

$$
\begin{aligned}
& A_{2}=x(0)+x^{\prime}(0) T+\frac{A_{1} T^{2}}{2}+\frac{B_{1} T^{3}}{6}+\int_{0}^{T} \frac{(T-s)^{3}}{6} F(s) d s \\
& B_{2}=x^{\prime}(0)+A_{1} T+\frac{B_{1} T^{2}}{2}+\int_{0}^{T} \frac{(T-s)^{3}}{2} F(s) d s
\end{aligned}
$$

are gained. The solution of the above system yields the following equations,

$$
\begin{aligned}
& x(0)=A_{2}-T B_{2}+\frac{A_{1} T^{2}}{2}+\frac{B_{1} T^{3}}{3}+\int_{0}^{T} \frac{1}{6}(T-s)^{3}(2 T+s) F(s) d s, \\
& x^{\prime}(0)=B_{2}-A_{1} T-\frac{B_{1} T^{2}}{2}-\int_{0}^{T} \frac{(T-s)^{2}}{6} F(s) d s .
\end{aligned}
$$

If we put (16) in (15), in that case we acquire

$$
\begin{aligned}
x(t) & =A_{2}-T B_{2}+\frac{A_{1} T^{2}}{2}+\frac{B_{1} T^{3}}{3}+\int_{0}^{T} \frac{(T-s)^{2}(2 T+s)}{6} F(s) d s+\left(B_{2}-A_{1} T-\frac{B_{1} T^{2}}{2}\right) \\
& -\int_{0}^{T} \frac{t(T-s)^{2}}{2} F(s) d s+\frac{A_{1} T^{2}}{2}+\frac{B_{1} T^{3}}{6}+\int_{0}^{t} \frac{(t-s)^{3}}{6} F(s) d s
\end{aligned}
$$


or

$$
\begin{aligned}
x(t) & =A_{2}-T B_{2}+\frac{A_{1} T^{2}}{2}+\frac{B_{1} T^{3}}{3}+\left(B_{2}-A_{1} T-\frac{B_{1} T^{2}}{2}\right) t+\frac{A_{1} T^{2}}{2}+\frac{B_{1} T^{3}}{6} \\
& +\int_{0}^{T}\left[\frac{(T-s)^{2}(2 T+s)}{6}-\frac{t(T-s)^{2}}{2}\right] F(s) d s+\int_{0}^{t} \frac{(t-s)^{3}}{6} F(s) d s
\end{aligned}
$$

for this reason, when we think $F(t)=f(t)-a(t) x$,

$$
\begin{aligned}
x(t) & =A_{2}-T B_{2}+\frac{A_{1} T^{2}}{2}+\frac{B_{1} T^{3}}{3}+\left(B_{2}-A_{1} T-\frac{B_{1} T^{2}}{2}\right) t+\frac{A_{1} T^{2}}{2}+\frac{B_{1} T^{3}}{6} \\
& +\int_{0}^{T}\left[\frac{(T-s)^{2}(2 T+s)}{6}-\frac{t(T-s)^{2}}{2}\right] f(s) d s+\int_{0}^{t} \frac{(t-s)^{3}}{6} f(s) d s \\
& -\int_{0}^{T}\left[\frac{(T-s)^{2}(2 T+s)}{6}-\frac{t(T-s)^{2}}{2}\right] a(s) x(s) d s-\int_{0}^{t} \frac{(t-s)^{3}}{6} a(s) x(s) d s,
\end{aligned}
$$

here

$$
\begin{aligned}
h(t) & =A_{2}-T B_{2}+\frac{A_{1} T^{2}}{2}+\frac{B_{1} T^{3}}{3}+\left(B_{2}-A_{1} T-\frac{B_{1} T^{2}}{2}\right) t+\frac{A_{1} T^{2}}{2}+\frac{B_{1} T^{3}}{6} \\
& +\int_{0}^{T}\left[\frac{(T-s)^{2}(2 T+s)}{6}-\frac{t(T-s)^{2}}{2}\right] f(s) d s+\int_{0}^{t} \frac{(t-s)^{3}}{6} f(s) d s
\end{aligned}
$$

we get

$$
x(t)=h(t)-\int_{0}^{T}\left[\frac{(T-s)^{2}(2 T+s)}{6}-\frac{t(T-s)^{2}}{2}\right] a(s) x(s) d s-\int_{0}^{t} \frac{(t-s)^{3}}{6} a(s) x(s) d s .
$$

The equation (17) is known as linear Volterra Fredholm integral equation where Fredholm operator has degenerated kernel. Let we define

$$
\begin{aligned}
V x & \equiv-\int_{0}^{t} \frac{(t-s)^{3}}{6} a(s) x(s) d s \\
F_{1} x & \equiv-\int_{0}^{T}\left[\frac{(T-s)^{2}(2 T+s)}{6}\right] a(s) x(s) d s \\
F_{2} x & \equiv \int_{0}^{T}\left[\frac{(T-s)^{2}}{2}\right] a(s) x(s) d s .
\end{aligned}
$$

So, the equation (17) can be arranged

$$
x(t)=h(t)+V x+F_{1} x+t F_{2} x
$$

by the reason of $F_{1} x, F_{2} x$ Fredholm and $V_{x}$ Volterra operators, respectively. Thereby, the problem (1)-(3) will be equivalent to the integral equation (18) [6].

\section{The generalized successive approximation method for elasticity problem}

To obtain the approximation of Volterra-Fredholm integral equation (18), we can use the following formula

$$
x_{n}(t)=h(t)+V x_{n-1}+F_{1} x_{n-1}+t F_{2} x_{n-1},(n=1,2, \cdots)
$$


here $h(t)=x_{0}(t)$ is known as discretionary and continuous function.

By solving the linear Volterra-Fredholm integral equation can calculate the approximation $x_{n}(t)$,

$$
y(t)=\tilde{h}(t)+F_{1} y+t F_{2} y
$$

where (20) has a degenerated kernel and has a solution

$$
y(t)=\tilde{h}(t)+C_{1}+t C_{2}
$$

In addition to that we can calculate the unknown terms $C_{1}$ and $C_{2}$ by solving the following linear equation system

$$
\begin{aligned}
\left(1-F_{1} 1\right) C_{1}-\left(F_{1} t\right) C_{2} & =F_{1} \tilde{h} \\
-\left(F_{2} 1\right) C_{1}+\left(1-F_{2} t\right) C_{2} & =F_{2} \tilde{h}
\end{aligned}
$$

If we assume the determinant of the coefficient matrix of (22) is not zero, namely

$$
\begin{aligned}
\Delta & =\left(1-F_{1} 1\right)\left(1-F_{2} t\right)-\left(F_{1} t\right)\left(F_{2} 1\right) \\
& =\left(1+\frac{1}{6} \int_{0}^{T}(T-s)^{2}(2 T+s) a(s) d s\right)\left(1-\frac{1}{2} \int_{0}^{T} s(T-s)^{2} a(s) d s\right) \\
& +\frac{1}{12}\left(\int_{0}^{T} s(T-s)^{2}(2 T+s) a(s) d s\right)\left(\int_{0}^{T}(T-s)^{2} a(s) d s\right) \neq 0 .
\end{aligned}
$$

We can calculate $C_{1}$ and $C_{2}$ as follows

$$
\begin{aligned}
& C_{1}=\frac{1}{\Delta}\left[\left(F_{1} \tilde{h}\right)\left(1-F_{2} t\right)+\left(F_{1} t\right)\left(F_{2} \tilde{h}\right)\right] \\
& C_{2}=\frac{1}{\Delta}\left[\left(1-F_{1} 1\right)\left(F_{2} \tilde{h}\right)+\left(F_{1} \tilde{h}\right)\left(F_{2} 1\right)\right]
\end{aligned}
$$

If we put into place $C_{1}$ and $C_{2}$ into the equation (21) we achieve the solution of the equation (20) as

$$
y(t)=\tilde{h}+\frac{1}{\Delta}\left[1-F_{2} t+t F_{2} 1\right]\left(F_{1} \tilde{h}\right)+\frac{1}{\Delta}\left[\left(1-F_{1} 1\right) t+F_{1} t\right]\left(F_{2} \tilde{h}\right) .
$$

To get the approximation of $x_{n}(t)$ we can use (19) and the equality $\tilde{h}(t)=h(t)+V x_{n-1}$ thus it yields the following approximation formula,

$$
x_{n}(t)=h^{*}(t)+\frac{1-F_{2} t+t F_{2} 1}{\Delta} F_{1} V x_{n-1}+\frac{\left(1-F_{1} 1\right) t+F_{1} t}{\Delta} F_{2} V x_{n-1}+V x_{n-1}
$$

here

$$
h^{*}(t)=h(t)+\frac{1-F_{2} t+t F_{2} 1}{\Delta} F_{1} h+\frac{\left(1-F_{1} 1\right) t+F_{1} t}{\Delta} F_{2} h
$$

To guarantee that the approximations of $x_{n}(t)$ is convergent to the solution of the problem (1-3), the following linear operator

$$
A(x)=\frac{1-F_{2} t+t F_{2} 1}{\Delta} F_{1} V x+\frac{\left(1-F_{1} 1\right) t+F_{1} t}{\Delta} F_{2} V x+V x
$$

must satisfy the inequalities

$$
\|A(x)\| \leq \beta\|x\|
$$




$$
\beta=\left(\frac{\left|1-F_{2} t\right|+T\left|F_{2} 1\right|}{6|\Delta|} \int_{0}^{T}(T-s)^{2}(2 T+s)|a(s)| d s+\frac{T\left|1-F_{1} 1\right|+\left|F_{1} t\right|}{2|\Delta|} \int_{0}^{T}(T-s)^{2}|a(s)| d s+1\right) \Theta<1 .
$$

where $\Theta=\left(\frac{1}{6} \int_{0}^{T}(T-s)^{3}|a(s)| d s\right)$. The convergence speed of the above approximations satisfies the inequalities

$$
\left\|x_{n}-x\right\| \leq \beta^{n}\left\|x_{0}-x\right\|
$$

or

$$
\left\|x_{n}-x\right\| \leq \frac{\beta^{n}}{1-\beta}\left\|x_{1}-x_{0}\right\|
$$

\section{Padé series}

The Padé series is defined as follows.

$$
a_{0}+a_{1} x+a_{2} x^{2}+\cdots=\frac{p_{0}+p_{1} x+\cdots+p_{M} x^{M}}{1+q_{1} x+\cdots+q_{L} x^{L}}
$$

After we multiply both sides of (26) by the denominator of right side of (26) and compare to the coefficients of both sides in (26). We obtain following equations

$$
\begin{aligned}
& a_{l}+\sum_{k=1}^{M} a_{l-k} q_{k}=p_{l},(l=0, \cdots, M) \\
& a_{l}+\sum_{k=1}^{L} a_{l-k} q_{k}=0,(l=M+1, \cdots, M+L) .
\end{aligned}
$$

By solving the linear equation in (28), we can acquire the values of $q_{k},(k=1, \cdots, L)$. Furthermore by substituting $q_{k}$ into (26), we obtain the values of $p_{k},(l=0, \cdots, M)[1,2]$.

\section{Test problem}

Let think the following elasticity problem with homogeneous boundary conditions and elasticity $a(t)=1$. According to these, the BVP can be given as

$$
\left\{\begin{array}{l}
\frac{d^{4} x}{d t^{4}}+x=t^{2} \\
x^{\prime \prime}(0)=0, x^{\prime \prime \prime}(0)=0 \\
x(1)=0, x^{\prime}(1)=1
\end{array}\right.
$$

If we calculate the approximate solution of (29) by using "The Generalized Successive Approximation" given in (24) we arrive at a solution as follows

$$
x_{3}(t)=0.000002 t^{9}-0.000022 t^{8}+0.002777 t^{6}-0.007242 t^{5}+0.037593 t^{4}+0.869141 t-0.902233
$$

This is the approximate solution of the problem (29) with the determinant value $\Delta=1.083680556 \neq 0$. 
Furthermore the solution of $x_{3}(t)$ can be transformed into Padé series as follows

$$
[7 / 6]=\frac{\left(\begin{array}{l}
-0.9022336418+0.8562379857 t+0.008166896781 t^{2}+0.004239419663 t^{3} \\
+0.03726935617 t^{4}-0.006513660254 t^{5}+0.002849747602 t^{6}
\end{array}\right)}{\left(\begin{array}{l}
1+0.01430226793 t+0.004725831968 t^{2} \\
-0.0001463043916 t^{3}+0.0002178515553 t^{4}-0.000002415627288 t^{5}
\end{array}\right)}
$$

Table 1: The comparison of the generalized successive approximation with the exact solution on the interval $[0,1]$.

\begin{tabular}{ccc|c}
\hline$t_{i}$ & $x\left(t_{i}\right)$ Exact Sol. & $x_{3}\left(t_{i}\right)_{[7 / 6]}$ & $\left.x\left(t_{i}\right)-x_{3}\left(t_{i}\right)_{[7 / 6]}\right]$ \\
\hline 0 & -0.9024272572 & -0.90223364180000 & 0.00019361540000 \\
0.1 & -0.8154922514 & -0.81531575450000 & 0.00017649690000 \\
0.2 & -0.7285066050 & -0.72834723770000 & 0.00015936730000 \\
0.3 & -0.6413443218 & -0.64120212250000 & 0.00014219930000 \\
0.4 & -0.5538022241 & -0.55367727310000 & 0.00012495100000 \\
0.5 & -0.4656036731 & -0.46549610760000 & 0.00010756550000 \\
0.6 & -0.3764003272 & -0.37631035510000 & 0.00008997210000 \\
0.7 & -0.2857719684 & -0.28569987650000 & 0.00007209190000 \\
0.8 & -0.1932244274 & -0.19317059350000 & 0.00005383390000 \\
0.9 & -0.0981856585 & -0.09815055403000 & 0.00003510447000 \\
1.0 & 0.0000000000 & +0.00001580208197 & 0.00001580208197 \\
\hline
\end{tabular}

\section{Conclusion}

The aim of this paper is to construct an approximate solution of the equation (9) which is elastic ground problem with variable coefficients. In Table 1 the solution of (29) is seen in detailed. The numerical outputs in the Table $\mathbf{1}$ show us that the approximate solution is very close to the exact solutions of (29).

\section{Competing interests}

The authors declare that they have no competing interests.

\section{Authors' contributions}

All authors have contributed to all parts of the article. All authors read and approved the final manuscript.

\section{References}

[1] E. Celik and M. Bayram, On the Numerical Solution of Differential-Algebraic Equation by Padé Series, Applied Mathematics and Computation, 137(2003) 151-160.

[2] E. Celik, E. Karaduman and M. Bayram, A Numerical Method to Solve Chemical Differential-Algebraic Equations, International Journal of Quantum Chemistry, 89(2002) 447-451.

[3] A. Aykut, E. Celik,and M. Bayram, The Ordinary Successive Approximations Method and Padé Approximants for Solving a Differential Equation with Variant Retarded Argument, Applied Mathematics and Computation, 2002, AMC 7569.

[4] E. Celik, A. Aykut and M. Bayram, The Modified Two-Sided Approximations Method and Padé Approximants for Solving a Differential Equation with Variant Retarded Argument, Applied Mathematics and Computation, 2002, AMC 7566.

[5] Francis B. Hildebrand. Methods of applied Mathematics (second edition), prentice-Hall, inc., New Jersey. 1965.

[6] E. Celik and M. Bayram, The basic successive substitute approximations Method and Padé Approximantions to solve the elasticity problem of settled of the wronkler ground with variable coefficients, Applied Mathematics and Computation, 154(2004) 495-505. 